\title{
Observational evidence for new instabilities in the midlatitude $E$ and $F$ region
}

\author{
David L. Hysell ${ }^{1}$, Miguel Larsen ${ }^{2}$, and Michael Sulzer ${ }^{3}$ \\ ${ }^{1}$ Department of Earth and Atmospheric Sciences, Cornell University, Ithaca, New York, USA \\ ${ }^{2}$ Department of Physics and Astronomy, Clemson University, Clemson, South Carolina, USA \\ ${ }^{3}$ Arecibo Observatory, Arecibo, Puerto Rico \\ Correspondence to: David L. Hysell (david.hysell@ cornell.edu)
}

Received: 19 May 2016 - Revised: 8 September 2016 - Accepted: 16 October 2016 - Published: 3 November 2016

\begin{abstract}
Radar observations of the $E$ - and $F$-region ionosphere from the Arecibo Observatory made during moderately disturbed conditions are presented. The observations indicate the presence of patchy sporadic $E\left(E_{\mathrm{s}}\right)$ layers, medium-scale traveling ionospheric disturbances (MSTIDs), and depletion plumes associated with spread $F$ conditions. New analysis techniques are applied to the dataset to infer the vector plasma drifts in the $F$ region as well as vector neutral wind and temperature profiles in the $E$ region. Instability mechanisms in both regions are evaluated. The mesosphere-lower-thermosphere (MLT) region is found to meet the conditions for neutral dynamic instability in the vicinity of the patchy $E_{\mathrm{S}}$ layers even though the wind shear was relatively modest. An inversion in the MLT temperature profile contributed significantly to instability in the vicinity of one patchy layer. Of particular interest is the evidence for the conditions required for neutral convective instability in the lower-thermosphere region (which is usually associated with highly stable conditions) due to the rapid increase in temperature with altitude. A localized $F$-region plasma density enhancement associated with a sudden ascent up the magnetic field is shown to create the conditions necessary for convective plasma instability leading to the depletion plume and spread $F$. The growth time for the instability is short compared to the one described by Perkins (1973). This instability does not offer a simple analytic solution but is clearly present in numerical simulations. The instability mode has not been described previously but appears to be more viable than the various mechanisms that have been suggested previously as an explanation for the occurrence of midlatitude spread $F$.
\end{abstract}

Keywords. Ionosphere (midlatitude ionosphere)

\section{Background}

The relative importance of plasma and neutral drivers in the midlatitude ionosphere is being debated in the contexts of sporadic $E\left(E_{\mathrm{S}}\right)$ layers, medium-scale traveling ionospheric disturbances (MSTIDs), and plasma density irregularities associated with midlatitude spread $F$. This paper informs the debates with observations from the Arecibo Radio Observatory. Patchy $E_{\mathrm{s}}$ layers, MSTIDs, and midlatitude spread $F$ occurred during the night of the 11 to the morning of 12 July 2015, over Arecibo during moderately disturbed, low solar flux conditions. The midnight collapse occurred as well. The event complements one reported on by Hysell et al. (2014b) recently which also exhibited these phenomena, albeit with important differences. These observations represent the most complete set of ground-based plasma and neutral state parameters available and are crucial for ranking the different mechanisms that could be responsible for the ionospheric irregularities, particularly at night.

Midlatitude sporadic $E\left(E_{\mathrm{s}}\right)$ ionization layers have been affecting communications since the earliest days of radio (see reviews by Layzer, 1962; Whitehead, 1972; Whitehead, 1989; Mathews, 1998). The dense metallic layers reflect, refract, diffract, and scatter radio signals, facilitating some radio applications and inhibiting others. While the layers can occur on continental spatial scales, the irregularities within that cause coherent radar scatter to extend down to meter scales or less. Deployments of coherent scatter radars in the modern era also highlighted important intermediatescale structuring in the layers (Riggin et al., 1986; Yamamoto et al., 1991, 1992). The coherent echoes have been termed 
"quasiperiodic" or QP because of periodicities in their rangetime-intensity representations.

Rocket experiments have shown that QP echoes come from patchy sporadic $E$ layers accompanied by strong polarization electric fields and strong neutral wind shear (e.g., Fukao et al., 1998; Larsen et al., 1998; Yamamoto et al., 2005; Bernhardt et al., 2005, and references therein). Studies using radar interferometry and imaging have shown that the echoes and underlying patchiness tend to be organized along fronts (e.g., Chu and Wang, 1997; Hysell et al., 2002; Saito et al., 2006). The fronts propagate with periods of 5-10 min, wavelengths of a few tens of kilometers, and directions preferentially toward the southwest in the Northern Hemisphere, although directions can vary significantly within and between events. The polarization electric fields spanning the fronts are often large enough to excite Farley-Buneman instability (Haldoupis and Schlegel, 1994), but field-aligned irregularities (FAIs) exist throughout the patchy layers even when the condition for Farley-Buneman instability is not met. Irregularities often come in bursts lasting about $1 \mathrm{~h}$.

Sporadic $E$ layer structuring leading to QP echoes is sometimes attributed to gravity waves (Woodman et al., 1991; Didebulidze and Lomidze, 2010; Chu et al., 2011) or an $E_{\mathrm{s}}$-layer plasma instability forced by neutral wind shear (Cosgrove and Tsunoda, 2002, 2004). The billowy appearance of the layers in incoherent scatter radar observations like those presented by Miller and Smith (1978) and Smith and Miller (1980) points to neutral shear (dynamical) instability as the cause (Larsen, 2000; Bernhardt, 2002; Hysell et al., 2004; Bernhardt et al., 2006; Larsen et al., 2007; Hysell et al., 2009). This premise is consistent with results from Larsen (2002) and Hecht et al. (2004), and others which suggest that the mesosphere lower thermosphere region is frequently dynamically if not convectively unstable.

Hysell et al. (2012) combined coherent and incoherent scatter radar measurements in a common volume to test the premise. They solved a boundary value problem (the Miles and Howard problem: Miles, 1961; Howard, 1961) for neutral dynamic instability, incorporating neutral wind profiles measured at Arecibo. The fastest-growing eigenmodes were found to have wavelengths, frequencies, and propagation directions comparable to the fronts in radar imagery from a coherent scatter radar on St. Croix. The $e$-folding time for the most unstable mode was only about $1 \mathrm{~min}$, and the instability was seen to be robust. That neutral rolls could be responsible for intermediate-scale $E_{\mathrm{s}}$-layer structuring is furthermore consistent with green-line optical imagery from Arecibo, which sometimes shows waves at least superficially similar to those seen in the coherent scatter radar imagery (see, e.g., Larsen et al., 2007).

Subsequently, Hysell et al. (2013) simulated plasma instability in patchy $E_{\mathrm{S}}$ layers produced by the aforementioned mechanism. The 3-D simulation code was unique in that it did not assume equipotential magnetic field lines, which is essential for studying ionospheric drift waves, for example.
A fast-growing class of collisional drift waves was found to emerge from polarized, patchy layers. These waves, which had kilometer scales, were similar to waves found in $E_{\mathrm{S}}$ layers using a high-resolution observing mode at Arecibo (Hysell et al., 2013). These transitional-scale waves were interpreted as being the primary plasma waves necessary to drive the meter-scale FAIs detected by the coherent scatter radar. Field-aligned currents played an essential role in the growth of the waves.

Arecibo data show that patchy $E_{\mathrm{s}}$ layers sometimes occur in conjunction with medium-scale traveling ionospheric disturbances (MSTIDs) and, more rarely, with midlatitude spread $F$ (see Behnke, 1979 for early Arecibo MSTID observations). MSTIDs are wavelike variations in the $F$-region ionization that exhibit periods of the order of $1 \mathrm{~h}$, wavelengths of hundreds of kilometers, and propagation speeds of $\sim 100 \mathrm{~m} \mathrm{~s}^{-1}$. They propagate mainly southwestward in the Northern Hemisphere. They are electrically polarized (Shiokawa et al., 2003) and represent the predominant ionospheric irregularities at middle latitudes. Miller et al. (2009) have furthermore shown evidence that traveling ionospheric disturbances (TIDs) propagating to low latitudes can instigate equatorial spread $F$, something Krall et al. (2011) have been able to reproduce in simulations.

Suzuki et al. (2009) showed that small-scale FAIs are also embedded in the phases of MSTIDs as if driven by the associated polarization electric fields under gradient drift instability (see also Otsuka et al., 2009; Ogawa et al., 2009). Small-scale field-aligned irregularities have indeed been detected at the phase nodes of MSTIDs observed in GPS-TEC (total electron content) maps in one event and in the troughs of MSTIDs observed in all-sky imagery in another. The irregularities give rise to spreading in ionogram traces and consequently to midlatitude spread $F$ by definition. However, the term "midlatitude spread $F$ " usually refers to the results of plasma convective instability in the midlatitude $F$ region which is morphologically similar to equatorial spread $F$ (ESF).

The plasma convective instability responsible for equatorial spread $F$ grows from perturbations to an equilibrium force balance between gravity and the $\boldsymbol{J} \times \boldsymbol{B}$ force due to Pedersen currents at low latitudes. The perturbation can be thought of as vertical displacements of slabs of plasma in planes parallel to the magnetic meridian plane. Since quasineutrality guarantees that the zonal Pedersen current be continuous in this case, perturbations in the weights of the slabs cannot be balanced, and so denser slabs descend, while less dense slabs ascend. Where the stratification is unstable, descending slabs get heavier, ascending ones lighter, and instability results. The instability is similar to the RayleighTaylor instability in neutral fluids except that inertia need not play an important role in the ionospheric case.

Krall et al. (2010) found that depleted plasma slabs or "bubbles" ascend under the aforementioned conditions until the magnetic flux-tube-integrated ion mass density matches 
that of the background. In their simulations, bubbles stopped rising at magnetic apex altitudes between 1200 and $1600 \mathrm{~km}$ where the stratification became stable. While this figure depends on background conditions in nature, bubbles would have to rise considerably higher to impinge on the ionosphere above Arecibo and higher than they have been observed rising over Jicamarca, even during strongly disturbed periods.

Perkins (1973) considered a generalization of the plasma convective instability responsible for ESF that permits something like it to operate at middle latitudes. The generalization is to consider field-aligned slabs of plasma that are tilted, i.e., from the vertical at the dip equator. In this case, quasineutrality guarantees that the current density is continuous in the direction normal to the slabs, but since this is no longer the zonal direction, the zonal current density giving rise to the component of $\boldsymbol{J} \times \boldsymbol{B}$ that balances gravity can vary between slabs. The tendency is for plasma on lower (higher) flux tubes with greater (lesser) flux-tube-integrated Pedersen conductivities to support smaller (larger) zonal Pedersen currents and to descend (ascend), leading to instability. The treatment was generalized later by Hamza (1999) to include the effects of background density gradients and neutral winds.

Perkins' instability is often cited as playing a role both in MSTIDs and midlatitude spread $F$. Numerical simulations have been able to reproduce realistic MSTIDs from random noise that conformed to the prescriptions of Perkin's linear theory until saturating (Yokoyama et al., 2008; Duly et al., 2014). In the latter case, the simulations were performed on complete flux tubes. Saturation occurred in that case after about $30 \mathrm{~min}$.

The long $e$-folding time of the Perkins instability, which is typically of the order of $1 \mathrm{~h}$ according to linear theory, together with its tendency to saturate at small amplitudes, suggests that other factors might be involved in MSTIDs and spread F. Kelley and Miller (1997) and Kelley (2011) argued that MSTIDs are induced merely by gravity waves rather than by Perkins instability and that they propagate in the direction for which dissipation is the weakest (see also Ogawa et al., 2009). This could be called the "Perkins stability" effect, whereby the midlatitude ionosphere exerts a damping force unless Perkin's criteria are met, in which case it is neutral. A long time history of airglow observations from Indonesia presented by Fukushima et al. (2012) supports the idea that MSTIDs are the result of gravity waves generated primarily by deep tropospheric convection rather than by plasma instability. Wind patterns in the troposphere were argued to be sufficient to account for the propagation directions observed.

Some lines of inquiry hold that $E$ - and $F$-region phenomena need to be considered together. Cosgrove and Tsunoda (2004) argued that the growth rate of the Perkins instability and $E_{\mathrm{s}}$-layer instabilities acting together is larger than that of either one acting alone (see also Tsunoda, 2006; Cosgrove, 2007). The hypothesis has been tested in a number of numerical investigations (e.g., Yokoyama et al., 2009). Otsuka et al.
(2008) and Otsuka et al. (2007) found a correlation between MSTIDs and intense $E_{\mathrm{s}}$ layers and $E_{\mathrm{s}}$-layer irregularities, respectively. Helmboldt et al. (2012) recently found evidence that sporadic $E$ layer irregularities and MSTIDs have related occurrence phenomenology in TEC data derived from the Very Large Array (VLA). Saito et al. (2007) also correlated MSTID and QP echo behavior over the MU radar in Japan but could not ascertain the causal relationship, i.e., which is the cause, which is the response?

The mechanism behind midlatitude spread $F$ (in the irregularity sense) and its relationship to MSTIDs and $E_{\mathrm{s}}$ layers are not well established. Irregularities accompany MSTIDs during the summer during periods of low solar activity (Fukao et al., 1991) but also emerge at other times when conditions are geomagnetically disturbed (Swartz et al., 2000). Midlatitude spread $F$ has also been associated with the steep bottom-side gradients that can form around the midnight collapse or rapid descent of the $F$ peak driven by meridional tidal winds and wind shears in the thermosphere (Crary and Forbes, 1986).

This paper informs the aforementioned debates with new, comprehensive observations from the Arecibo Radio Observatory. New analytic tools permit neutral diagnostics and stability analysis in the neutral mesosphere-lowerthermosphere (MLT) region. A new simulation code elucidates a heretofore neglected instability mechanism in the midlatitude $F$ region. Below, the data, tools, and simulation are presented and evaluated in a common context.

\section{Observations}

The period from 10 to 12 July 2015 was moderately disturbed, with the Kp index exceeding 4 for three contiguous $3 \mathrm{~h}$ periods at the start of 11 July. The F10.7 solar flux index was approximately 120 . Ionospheric irregularities were observed in turn over Arecibo on the evening of 11 July through the morning of 12 July.

The digisonde in San Juan, Puerto Rico, was detecting a sporadic $E$ layer with a blanketing frequency of $6.5 \mathrm{MHz}$ and a top frequency of $7.25 \mathrm{MHz}$ by $16: 15 \mathrm{LT}$ on 11 July 2015 . The gap between the two frequencies increased steadily thereafter. By 19:45 LT, the top frequency of what had become a patchy layer was approximately $9 \mathrm{MHz}$. The sporadic $E$ layer traces would remain strong and variable until about 03:00 LT the following morning. In addition, strong ionosonde spread $F$ emerged at about 23:30 LT. The spread $F$ was strongest through 01:30 LT the following morning and persisted until sunrise.

Arecibo observations for 11/12 July 2015, are shown in Fig. 1. For these experiments, data were collected using both the line feed and Gregorian feed systems. The former was pointed at zenith, and the latter was pointed at a $15^{\circ}$ zenith angle and scanned in azimuth between west and north. Dual-beam data such as these permit inferences about vector 

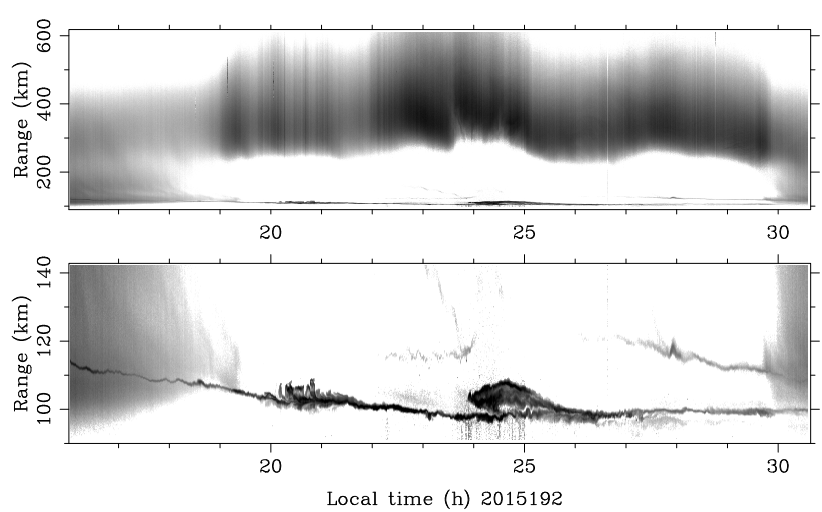

Figure 1. Incoherent scatter observed by the line feed system at the Arecibo Radio Observatory on 11/12 July 2015. Grayscales represent range-corrected power, which serves as a proxy for electron number density. The top panel shows $E$ - and $F$-region data, whereas the bottom panel shows $E$-region data in more detail.

plasma and neutral drifts in three spatial dimensions (see below).

Data were acquired using the coded long-pulse (CLP) mode developed by Sulzer (1986b). This represents a departure from synoptic experiments run in the recent past at Arecibo, which usually divided time between the CLP mode and a multi-frequency mode optimized for $F$-region plasma drift estimation (Sulzer, 1986a). The change was made to facilitate the acquisition of more plasma-line data. $F$-region plasma drifts can still be estimated from CLP data, albeit at a lower cadence. The cadence for the processed data shown here is once per minute.

Incoherent scatter (ion-line) power profiles acquired with the line feed system are shown in Fig. 1. The backscatter power has been corrected for range and serves as a proxy for electron density. The upper panel spans the $E$ and $F$ regions, whereas the lower panel presents an exploded view of the $E$ region.

Between 19:00 and 21:30LT, the $F$-region densities at all altitudes underwent quasiperiodic modulation with a dominant period of a little less than $1 \mathrm{~h}$. The phenomenon suggests the passage of medium-scale traveling ionospheric disturbances (MSTIDs).

Beginning about $30 \mathrm{~min}$ after midnight, the $F$ region fell rapidly. This is suggestive of the midnight collapse, a recurring dynamical feature in Arecibo datasets (e.g., Gong et al., 2012, and references therein). We will not focus attention on the midnight collapse but refer to it to establish context for some other dynamical events.

Starting $1 \mathrm{~h}$ before midnight, the height of the bottom side fell and then rose even more sharply. The sharp rise is also a recurring feature over Arecibo that can be regarded as preconditioning for eventual collapse. Just before midnight on this occasion, a narrow, depleted channel extending from the bottom side to the topside passed over the observatory. For a period of about $1 \mathrm{~h}$ after the passage of the depleted channel, the bottom-side $F$ region exhibited considerable structuring at scale sizes down to the finest that could be resolved by this observing mode. The depleted channel and the fine structure that followed were morphologically similar to what is observed at the magnetic equator during periods of equatorial spread $F$. Later in the paper, we will explicitly link the depleted channel to the large-density perturbation that preceded it.

Irregular layers in the $E$ region that appear concurrently are highlighted in the lower panel of Fig. 1. A sporadic $E$ layer was present through sunset on 11 July. After sunset, as very often occurs in the summer months, the sporadic $E$ layer persisted but became irregular. Between 20:00 and 21:30 LT, the layer became patchy and vertically developed. Its billowy appearance suggests a connection with neutral dynamic instability, as demonstrated by Bernhardt (2002). As mentioned above, coherent scatter radar observations in a common volume have shown that ionized patches like these are usually organized along elongated wavefronts with wavelengths of a few tens of kilometers (e.g., Hysell et al., 2012). In this case, the patchy layer was observed at about the same time as the MSTIDs which preceded it by about $1 \mathrm{~h}$. Note that previous investigations at Arecibo have not revealed a universal relationship between patchy sporadic $E$ layers and MSTIDs. In the event documented by Hysell et al. (2014b), for example, MSTIDs and patchy $E_{\mathrm{s}}$ layers occurred on the same night but were mutually exclusive in time. In the present case, the MSTIDs and $E_{\mathrm{S}}$ layers exhibit dissimilar periodicities.

Whereas the aforementioned feature was a typical example of sporadic $E$ layer morphology, the feature in evidence between 00:00 and 02:00 LT is unusual. The feature was more than $10 \mathrm{~km}$ thick at times and morphologically suggestive of a single roll. The feature was moreover precisely coincident with the elevation of the $F$ layer and the most intense period of midlatitude spread $F$.

Density profiles from the Gregorian feed (not shown) are substantially similar to Fig. 1 except for modulation in time due to azimuth swinging. The modulation is barely detectable in the $F$ region before 23:30 LT but severe thereafter. This indicates that horizontal fine structuring on the scale of the beam separation was largely absent before the emergence of spread $F$ and widespread afterward. The second $E_{\mathrm{S}}$-layer patch was also free of modulation, implying that the sporadic $E$ layer blob was relatively unstructured on these scales.

More comprehensive measurements and derived parameters are shown in Fig. 2. Panels a and b show line-of-sight plasma drift estimates from line feed data acquired in the $E$ and $F$ regions, respectively. The estimates are based on weighted averages of the phases of the first few lags of the incoherent scatter radar autocorrelation functions. Whereas the $E$-region estimates are range resolved, the $F$-region estimates are altitude averaged (to improve statistical confidence). Altitude variations in panel a reflect the varying in- 

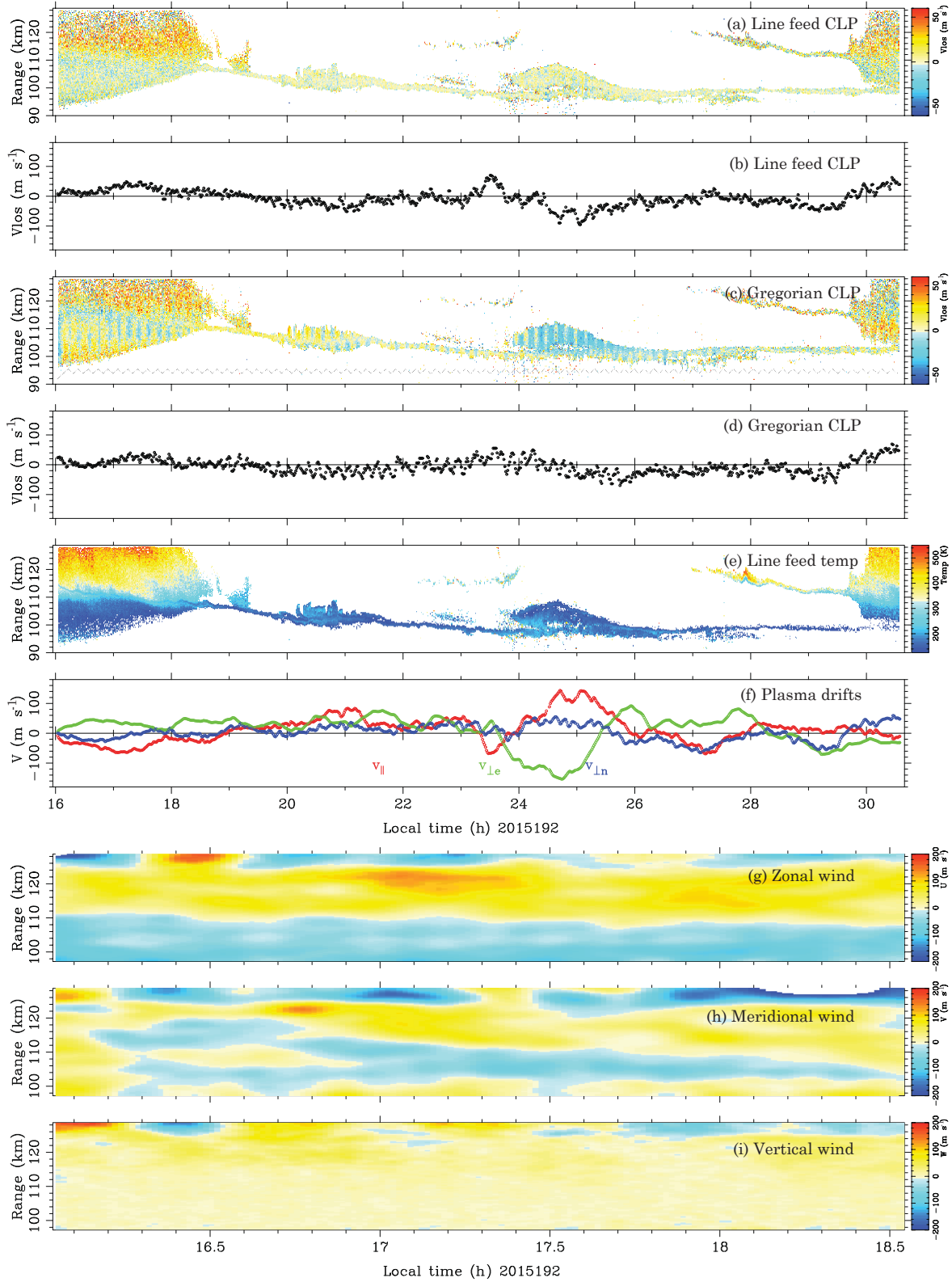

Figure 2. Detailed examination of the Arecibo dataset from 11/12 July 2015. The panels are described in the text.

fluence of winds, Pedersen drifts, and Hall drifts in different strata.

Similar remarks hold for panels c and d, which represent Gregorian feed (off-zenith) plasma drift estimates. Regular modulation in the drift estimates is a consequence of strong zonal flows combined with azimuth steering. Additional altitude variations in the Gregorian-feed drifts indicate shear flow.

Plasma temperature and composition are estimated from the autocorrelation functions using the procedure described by Hysell et al. (2014b). A simple composition model that allows for $\mathrm{O}^{+}, \mathrm{NO}^{+}$, and $\mathrm{O}_{2}^{+}$ions and used temperature- dependent rate coefficients is incorporated in the parameter fitting process iteratively. The resulting temperature estimates are shown in panel e. These estimates are expected to be valid in the sunlit $E$ region but not in the sporadic $E$ layers, which are reanalyzed differently below. Between 16:00 and 18:00 LT, there is the suggestion of significant dynamics and mixing in the $E$ layer, i.e., the isotherms are not vertically stratified.

Figure $2 \mathrm{f}$ presents $F$-region plasma drift estimates derived from the line feed and Gregorian line-of-sight drift measurements. Here, an inverse method incorporating a forward model of the (anisotropic) plasma mobility along with 

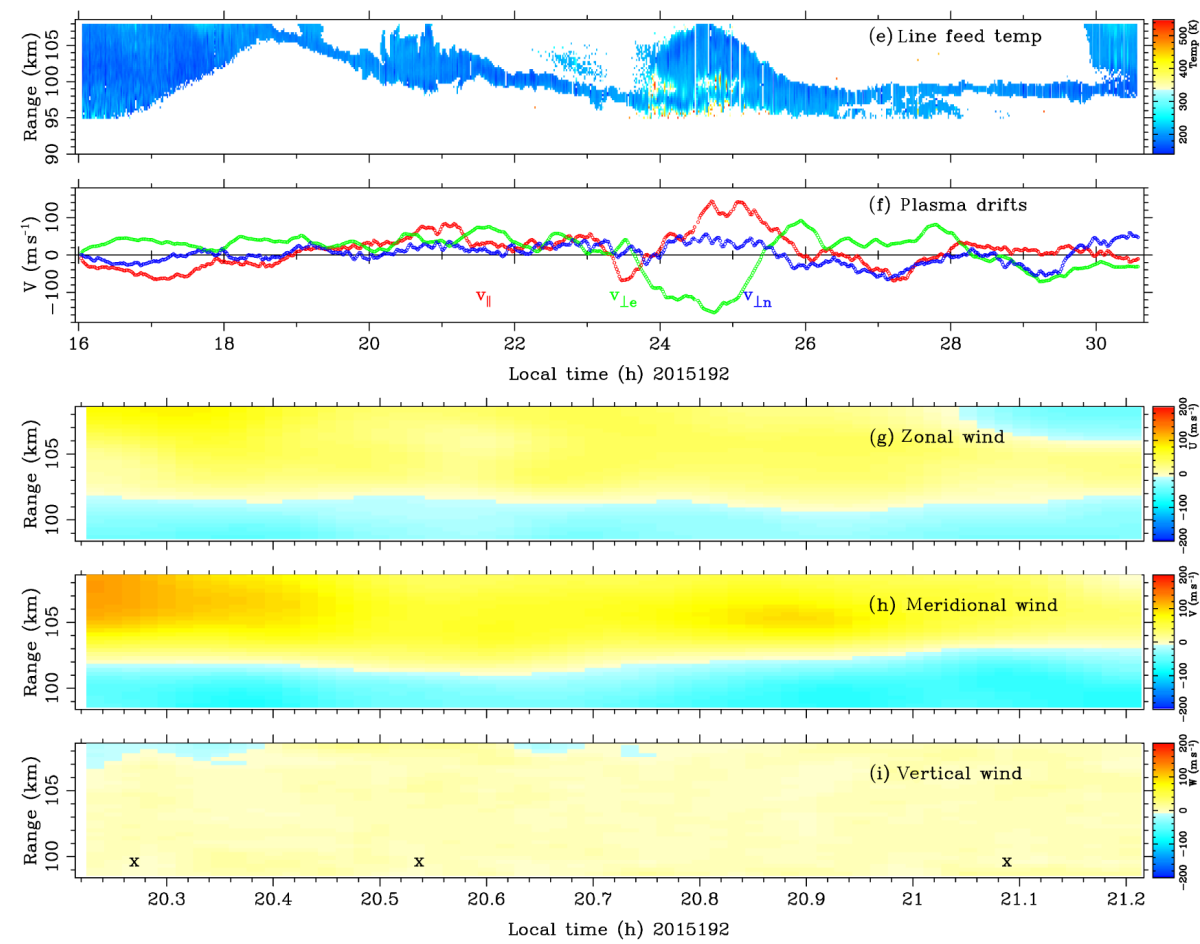

Figure 3. Detailed examination of the Arecibo dataset from 11/12 July 2015. The focus here is on the first patchy sporadic $E$ layer. The panels are described in the text. Note that panels (a) through (d) in Fig. 2 apply to the entire experiment and are not reproduced in Figs. 3 and 4 .

regularization is used to estimate drifts in the parallel-to$B$, perpendicular-east, and perpendicular-north directions, which are consistent with the available line-of-sight drifts while having minimal curvature. The method is described in detail in Hysell et al. (2014b) and is similar to that introduced by Sulzer et al. (2005). Two main features are present in the drifts. The first feature is a sharp ascent anti-parallel to $B$ peaking at 23:30 LT followed by a rapid descent along $B$ peaking broadly at 01:00 LT. The ascent followed a brief plunge in the $F$-layer height. The descent is consistent with the apparent midnight collapse of the $F$-layer height.

The second feature is rapid westward plasma advection between 23:30 and 01:30 LT. Notably, this is nearly coincident with the appearance of the sporadic $E$ layer blob.

Lastly, Fig. $2 \mathrm{~g}-\mathrm{i}$ show estimates of the zonal, meridional, and vertical winds in the sunlit $E$ region from 16:00 to 18:30 LT. The winds are also estimated using an inverse method that incorporates a forward model of the plasma mobility together with regularization and curvature minimization, as described by Hysell et al. (2014b). The electric fields deduced from the $F$-region analysis are assumed to be representative of the $E$ region as well and incorporated into the wind-estimation problem. Strong zonal and meridional shear is indicated, with the shear node being closely collocated with the thin sporadic $E$ layer. Atypically large vertical winds with a periodicity of about $1 \mathrm{~h}$ are also indicated in the highest altitudes at which the wind analysis could be carried out.

Figure 3 presents data in a manner similar to Fig. 2 but focuses on the first patchy sporadic $E$ layer. The incoherent scatter data were processed differently in two respects. First, no composition modeling was performed. Instead, the composition was assumed to be a combination of iron and magnesium ions, and temperature and composition were estimated through ordinary nonlinear least-squares fitting of the autocorrelation functions. Second, to accommodate the sparseness of the sporadic $E$ echoes in range, a global fitting strategy was used, similar to that described by Cabrit and Kofman (1996) (see also Hysell et al., 2009). In this strategy, state parameters for all altitudes are fit simultaneously, and an additional penalty for the curvature of the parameters with altitude is imposed. This produces parameter profiles that are consistent with all available data while varying gradually with altitude. Continuity in time is not enforced in the global fitting.

Figure $4 \mathrm{e}$ shows temperature profiles within the patchy sporadic $E$ layers, where the aforementioned methodology is expected to be applicable. (Ion temperatures are what have been measured, but we equate these with neutral temperatures; the ion cooling time due to nonresonant collisions with neutrals is much shorter than the timescale for modifying the ion temperature dynamically.) Temperatures within the first 

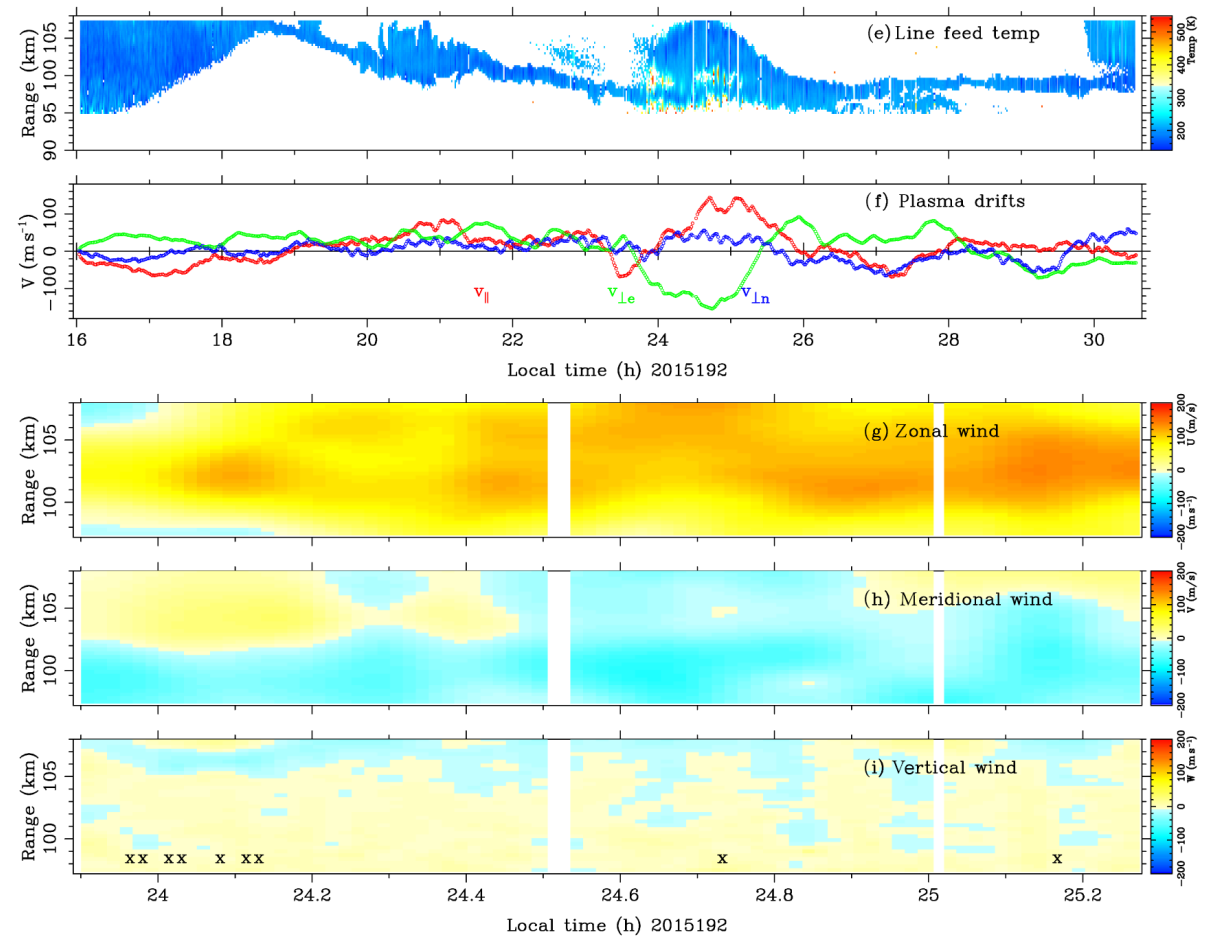

Figure 4. Detailed examination of the Arecibo dataset from 11/12 July 2015. The focus here is on the second patchy sporadic $E$ layer. Panels (e)-(i) are described in the text.

patch are almost uniform or slightly increasing with altitude. Within the second patch, however, the temperatures appear to decrease with altitude much of the time.

Panels g-i show wind estimates within the first patchy sporadic $E$ layer between 20:12 and 21:15 LT. The methodology used here was the same as described above and in Hysell et al. (2014b) except that conductivities for metallic ions based on collision frequency formulas found in Schunk and Nagy (2004) were utilized in the inversion. The panels reflect planar shear flow, with winds reversing from southwest at low altitudes to northeast at high altitudes. Vertical winds are very small, as is usually the case. The flows are consistent over time. Little turning shear is present.

Lastly, Fig. 4 focuses on the second patchy sporadic $E$ layer. The new information here is in panels $\mathrm{g}-\mathrm{i}$, which show the wind estimates between 23:55 and 01:15 LT. The winds are rapid and toward the southeast for the most part. Shear is evident but is not particularly strong except toward the end of the event. Vertical winds are again small.

Figures 5 and 6 present measurements within the first and second $E_{\mathrm{s}}$ layers (respectively) in still greater detail. The figures show the progression over time of the zonal $(U)$ and meridional $(V)$ winds. Different colors, progressing from black to red to green to blue, represent different times in the given intervals. The violet curves are models selected to represent typical profiles in analyses carried out in the next section of the paper. Also shown in Figs. 5 and 6 are temper- atures averaged over the intervals in question. Lines through the plotted points are sample variances. In the case of the first patch, there is little natural variability, and the sample variances reflect mainly statistical uncertainty in the measurement. Variability is greater in the second patch, and the sample variances are consequently larger.

The zonal and meridional winds are similar in shape in the first patch, meaning that the shear, which is significant, is planar as opposed to turning shear. The temperatures increase throughout the layer at a rate of $2-3 \mathrm{~K} \mathrm{~km}^{-1}$. The layer is convectively stable but possibly dynamically unstable. The case of the second layer is more unusual. The zonal and meridional wind profiles have rather different shapes. The shear is relatively modest except at first when $U$ varied strongly with altitude. Most remarkably, the temperature profile is inverted. Around $100 \mathrm{~km}$ altitude, the lapse rate is even comparable to the adiabatic lapse rate of $\sim 9.5 \mathrm{~K} \mathrm{~km}^{-1}$. This suggests the possibility even of convective instability and all but guarantees dynamic instability.

\section{Analysis}

We focus our analysis on two aspects of irregularities in the Arecibo dataset: the patchy sporadic $E$ layers and the spread $F$ plume. For the first, we analyze the possible role of neutral dynamic instability. For the second, we consider the possibil- 

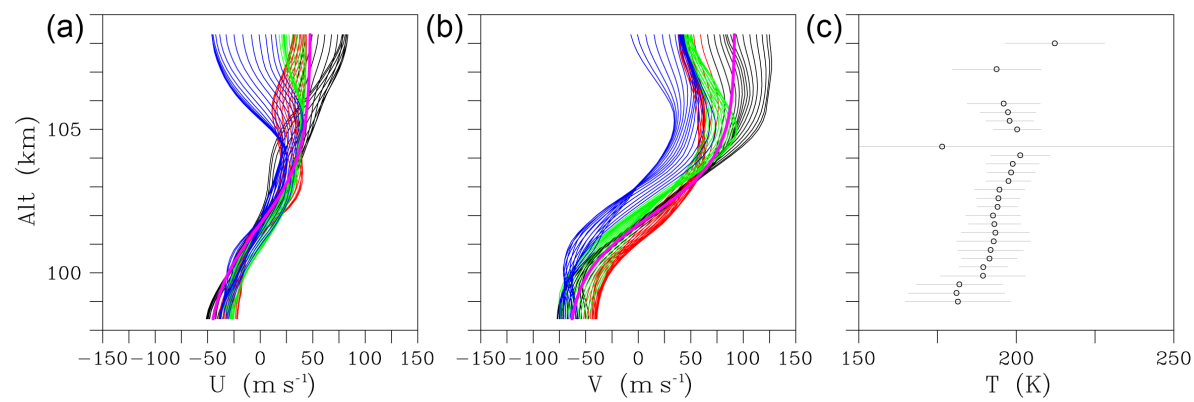

Figure 5. State-parameter estimates within the sporadic $E$ layer patch seen between 20:12 and 21:15 LT. Left: zonal winds. Middle: meridional winds. Right: temperatures. The change in color, from black to red to green to blue, indicates the passage of time. The violet curves are models reflecting conditions at 20:24 LT used in subsequent analysis.
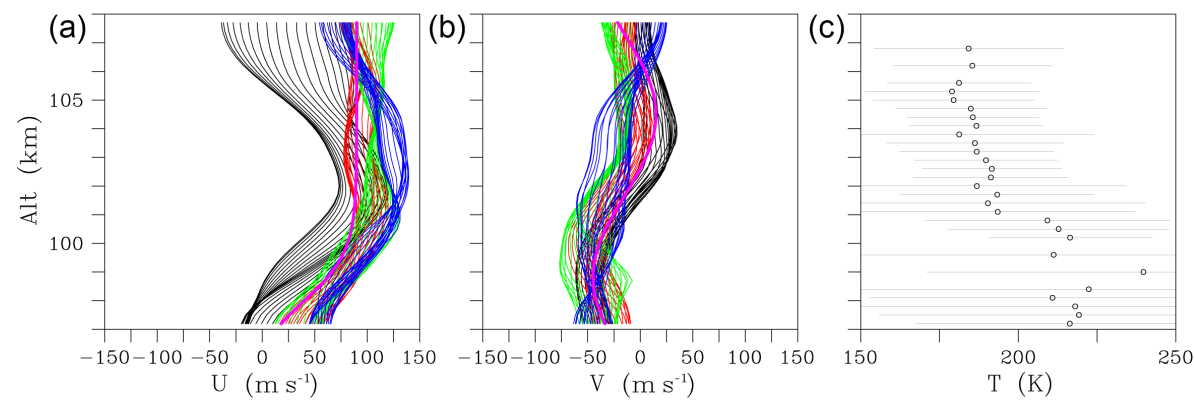

Figure 6. Same as Fig. 5 except for 23:50-01:20 LT. The violet curves are models reflecting conditions at 00:12 LT.

ity of plasma convective instability. These are the avenues of investigation with the most support in the available data.

\section{1 $E_{\mathrm{s}}$ layers}

We solve the Miles-Howard problem (Miles, 1961; Howard, 1961) to assess the dynamical stability of the MLT region in the vicinity of the two $E_{\mathrm{s}}$-layer patches observed over Arecibo. This is a boundary value problem that derives from the linearized equations of mass and momentum conservation in a vertically stratified, non-Boussinesq fluid. The inputs to the problem are horizontal wind profiles spanning the strata of interest and the local Brunt-Väisälä frequency. Dirichlet boundary conditions are imposed at the upper and lower altitude limits. The eigenvalue sought is the complex phase speed. Real solutions imply propagating waves, and imaginary solutions imply growing mode shapes. The problem is solved numerically using a relaxation method (Press et al., 1988). Details regarding the problem and its solution are given by Hysell et al. (2012).

Figure 7 shows results of the analysis for the first of the two $E_{\mathrm{S}}$-layer patches. For this analysis, the Brunt-Väisälä period is taken to be $5 \mathrm{~min}$. The model wind profiles used to represent the conditions in the patch are plotted in the lowerleft panel. The upper-left panel shows the minimum Richardson number $\left(R_{i}\right)$ for those profiles as well as the propagation angle (in the horizontal plane measured in degrees east of north) for which $R_{i}$ is a minimum. The curve suggests that a necessary condition for dynamic instability, viz., $R_{i}<\frac{1}{4}$, is satisfied, if just barely, in a narrow stratum.

The upper-right panel of the figure shows the growth rate for dynamical instability for different wave vectors. Here, $x$ and $y$ denote eastward and northward directions, respectively. Unstable solutions exist for northeast-southwest propagating modes with wavelengths of about $20 \mathrm{~km}$. The $e$ folding time for the fastest-growing modes is of the order of $15 \mathrm{~min}$. Marginal instability, limited by the stabilizing effect of buoyancy, is therefore indicated.

The three sets of curves superimposed in the upper-right panel are mode shapes calculated at the three points indicated. The mode shapes are concentrated close to the strata of maximum shear, implying only shallow mixing. The phase velocities for the unstable modes are small, as indicated by the lower-right panel in the figure. Rolls caused by the instability would be expected to drift with the winds at the shear node, which were small in this case.

Figure 8 shows the results of a similar analysis applied to the second $E_{\mathrm{s}}$-layer patch. In view of the lapse rate within this patch, which approaches the adiabatic rate over some spans of altitude, the Brunt-Väisälä period is taken to be very long for this analysis. The removal of the buoyancy stabilization gives rise to a number of families of fast-growing solutions that would not otherwise be present. The upper-right 

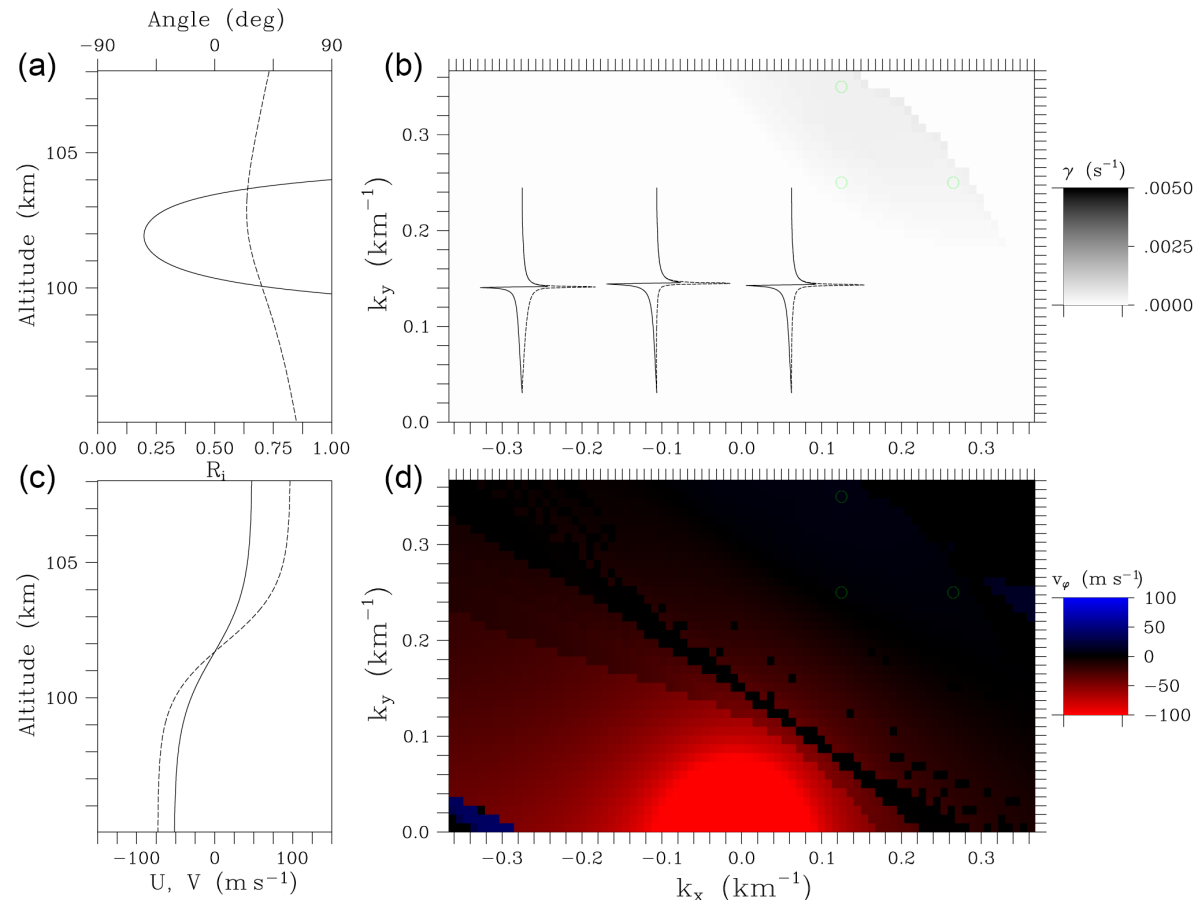

Figure 7. Eigenfunction analysis for wind profiles in first patchy $E_{\mathrm{S}}$ layer. (a) Minimum Richardson number parameter and propagation angle at which the minimum occurs. (b) Linear growth rate of the fastest-growing eigenmode with some representative mode shapes. (c) Zonal (solid) and meridional (dashed) wind profiles. (d) Phase speed of fastest-growing mode.
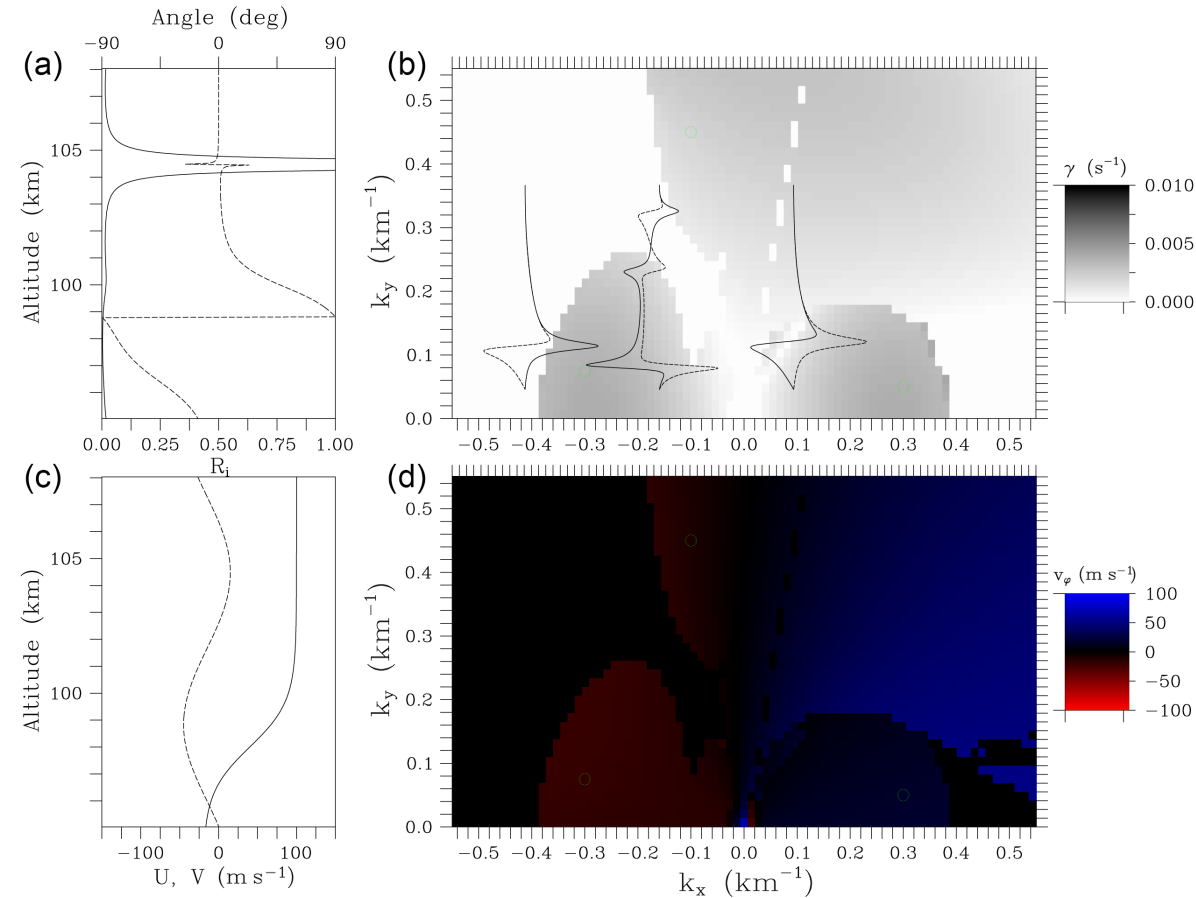

Figure 8. Same as Fig. 7 except for the second $E_{\mathrm{S}}$-layer patch. 
panel of the figure reveals growing modes propagating in all directions.

The fastest-growing waves have $e$-folding times of about $2 \mathrm{~min}$ and wavelengths of $20-30 \mathrm{~km}$. This is considerably shorter than predictions for $E_{\mathrm{S}}$-layer instability or its variants under the same forcing conditions (e.g., Cosgrove and Tsunoda, 2004; Tsunoda, 2006). The mode shapes for the most unstable waves span large vertical distances, implying the possibility of deep overturning, particularly in the case of waves propagating in the meridional directions. The predicted phase speeds for the fastest-growing modes are again small. Irregularities are expected to drift with the background winds, which are mainly eastward in this case.

\subsection{Spread $F$ plume}

The introductory discussion about midlatitude $F$-region plasma instability focused on mechanisms involving motions of planar slabs of ionization. Such mechanisms are easy to visualize, amenable to linear, local analysis, and were prime candidates for explaining midlatitude spread $F$ when it was first being observed at Arecibo. However, contemporary computational tools permit the exploration of a wider range of candidate mechanisms that can tap the free energy in the unstably stratified nighttime ionosphere.

Aside from the depletion plume observed at 23:30 LT, the most prominent feature in the $F$ region in Fig. 1 is the zone of enhanced ionization in the bottom side at about $300 \mathrm{~km}$ altitude that immediately preceded the plume. According to the $F$-region drift estimates in Fig. 2, this feature was accompanied by rapid, widespread motion of ionization up and down magnetic field lines. The displacement of ionization along the magnetic field lines at middle latitudes signifies a drastic redistribution of conductivity. The enhancement in Fig. 1 represents a bulge in field-line-integrated Pedersen conductivity with significant dynamical consequences. It could well have been the seed for instability and midlatitude spread $F$ onset.

We have used a 3-D numerical simulation to examine the effects of a conductivity bulge in the midlatitude ionosphere. The simulation code is a modified version of the one described by Hysell et al. (2014a). It evolves the number density of four ion species $\left(\mathrm{O}^{+}, \mathrm{O}_{2}^{+}, \mathrm{NO}^{+}\right.$, and $\left.\mathrm{H}^{+}\right)$in time, incorporating the effects of background electric fields, winds, pressure gradients, gravity, and recombination chemistry. Initial conditions for the plasma number density and composition are derived from a combination of empirical models. The code solves for the electrostatic potential fully in three dimensions by enforcing quasi-neutrality. The code is cast in tilted magnetic dipole coordinates (see Swisdak, 2006, for discussion). The simulation space encompasses the $E$ and $F$ regions in the Northern Hemisphere in the American sector and is bounded by $p \in[1.03,1.08], q \in[0,0.24]$, and $\phi \in[-2.0,2.0]$ where $q \equiv \cos \theta / r^{2}, p \equiv r / \sin ^{2} \theta, \theta$ is magnetic co-latitude, and $\phi$ is degrees longitude. The simulation space therefore spans a zonal distance of just over $400 \mathrm{~km}$ at the magnetic equator and $L$ shells from 1.03 to 1.08 .

For this simulation, a zonal wind profile with a hyperbolictangent profile shape drives eastward plasma drifts approaching $100 \mathrm{~m} \mathrm{~s}^{-1}$ at the top of the simulation volume. Note that the direction of the winds is immaterial and that the phenomena described below occur given eastward or westward winds and background plasma drifts. A constant background zonal electric field drives plasma ascent at $30 \mathrm{~m} \mathrm{~s}^{-1}$. Ascent in the plane perpendicular to $B$ is required for instability in the scenario depicted. The drifts in the $v_{\perp n}$ direction were positive most of the time in the hours before the appearance of the spread $F$ plume and so consistent with this assumption.

The left column of Fig. 9 depicts the initial conditions. The upper panel shows the plasma number density in the plane perpendicular to $B$ in the meridional midpoint of the simulation. Red, green, and blue tones represent molecular ions, atomic oxygen ions, and hydrogen ions, respectively. The panel reflects the addition of an ellipsoidal blob of enhanced density in the bottom side. The plasma density is doubled where the blob is densest.

The middle panel shows the current density in the same perpendicular-to- $B$ plane. Superimposed on the current density are equipotential contours in units of $\mathrm{kV}$. These are approximate streamlines of the transverse-to- $B$ flow. The streamlines indicate the well-known behavior of plasma dynamics in the vicinity of a Pedersen conductivity enhancement or blob. The effects are twofold. First the blob drifts with the zonal wind faster than the background plasma. Second, the blob descends in the background plasma frame of reference. Since the background plasma is ascending, the net effect is that the blob nearly maintains its altitude.

Since the transverse-to- $B$ flow is nearly incompressible, the plasma flow surrounding the blob is deflected around it. The resulting vertical motion perturbs the background plasma density gradient, producing depletions to the east and west of the blob. These depletions are subsequently prone to normal $\boldsymbol{E} \times \boldsymbol{B}$ instability. After $37.5 \mathrm{~min}$ of simulation time, the depletion on the leading edge of the enhancement exhibits vertical drifts in excess of $200 \mathrm{~m} \mathrm{~s}^{-1}$ in this simulation. The ascent rate moreover increases rapidly toward the end of the simulation as the depletion channel becomes more narrow and structured. Our simulation was terminated as the depletion propagated outside of the simulation space. In nature, the depletion would be expected to propagate well into the topside $F$ region.

We can estimate a growth rate for the instability by dividing the ascent speed of the depletion by the vertical density gradient scale length $L \sim 20 \mathrm{~km}$. The corresponding $e-$ folding time of $100 \mathrm{~s}$ is much shorter than can be expected from the Perkins instability or its variants.

The bottom panel in the right column of Fig. 9 shows the current density in the plane of the magnetic meridian. The particular plane shown here is coincident with the westward wall of the depletion. Very strong downward field-aligned 

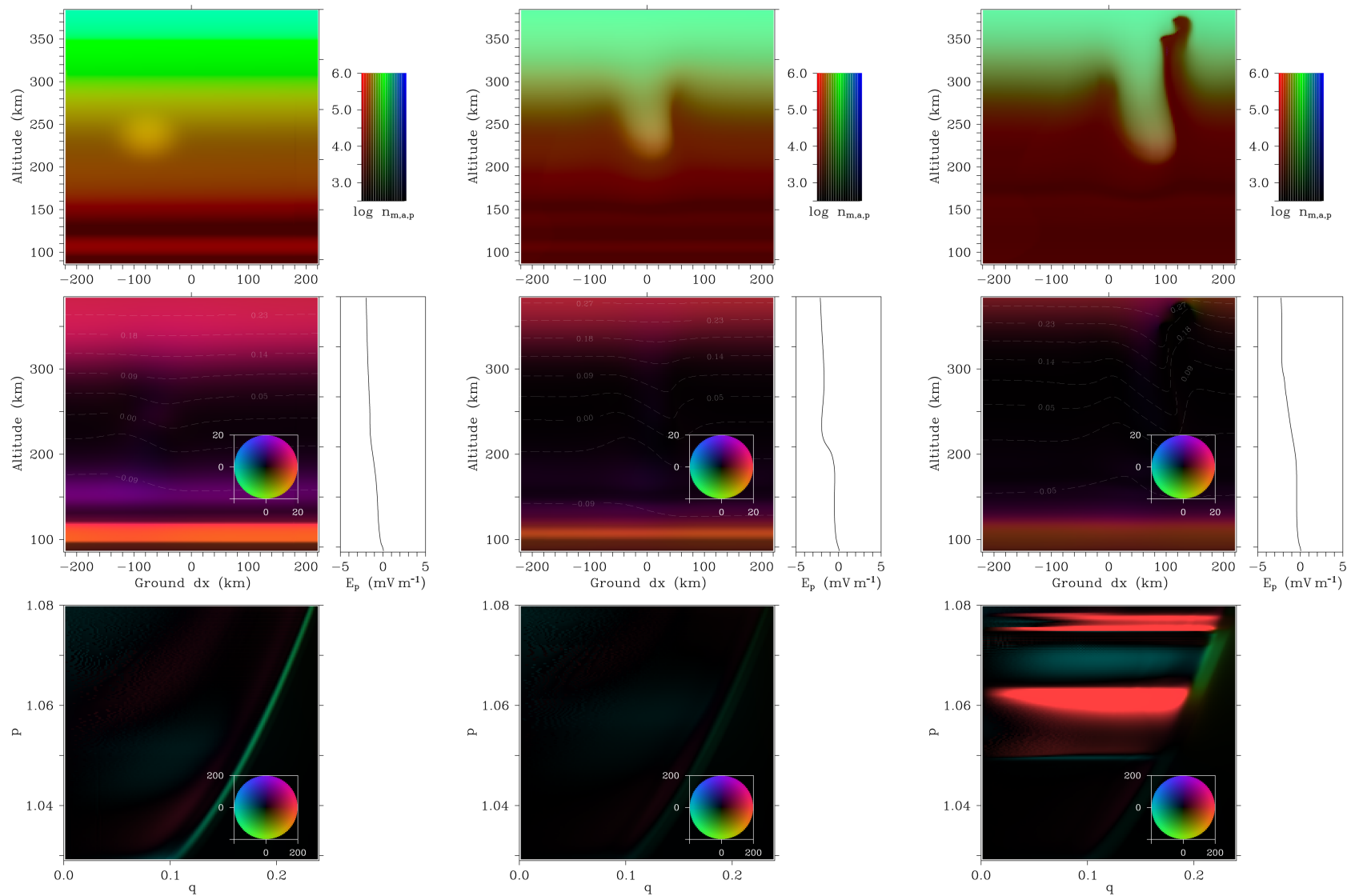

Figure 9. Numerical simulations of midlatitude spread $F$. The left, center, and right columns depict simulation times of 0,20 , and 37.5 min, respectively. The top panels of each column show plasma number density in the plane perpendicular to $B$ in the meridional center of the simulation. The colors indicate the abundances of molecular ions (blue), atomic oxygen ions (green), and protons (red). The middle panels show vector current density in the same plane according to the indicated color legend (full scale: $20 \mu \mathrm{A} \mathrm{m}{ }^{-2}$ ). For clarity, contributions from diamagnetic currents are not shown. Equipotential curves in kilovolt are superimposed. The bottom panels show current density in a magnetic meridional plane at a $50 \mathrm{~km}$ zonal ground distance according to the indicated color legend (full scale: $200 \mu \mathrm{A} \mathrm{m}{ }^{-2}$ ).

currents are seen to flow on the meridional edges of the depleted region. The currents close in the $E$ region. We can speculate that the currents would provide the free energy for secondary instabilities in sporadic $E$ layers sharing magnetic field lines with similar depletions in nature. Details regarding the secondary instabilities in question are provided by Hysell et al. (2013).

The spread $F$ event described by Hysell et al. (2014b) was similar to the one described here in several respects. It occurred between sunset and midnight and was preceded by a sharp, downward displacement of plasma along $B$ and an attendant density enhancement. The major difference was that the earlier event produced an ascending plume of enhanced rather than depleted plasma, something that is never observed at equatorial latitudes. We argue that the mechanism proposed here could account for that event if (1) the initial conductivity enhancement occurred in the topside and (2) the background plasma was descending rather than ascending. (These conditions were met in the earlier event.) In that case, the deflection of plasma around the blob would tend to pull dense ionization from the $F$ peak upward and push more rarefied plasma from the topside downward. The dense plasma would then continue to ascend in the topside and become unstable under the action of the westward background electric field. The phenomenology is almost symmetric with that observed in the present event albeit more slowly growing. Enhanced density plumes are inherently less unstable than depleted ones, and the corresponding mechanism is not as robust. Both mechanisms appear to be viable, however.

\section{Summary and conclusions}

We have presented observations from the Arecibo Observatory of the $E$ - and $F$-region ionosphere during moderately disturbed conditions. The observations exhibit the most common manifestations of space weather at middle latitudes: patchy $E_{\mathrm{s}}$ layers, MSTIDs, and spread $F$ depletions. Although the phenomena occurred at about the same time, clear 
connections between them are not obvious. Indeed, a conclusion of the paper is that there appear to be viable instability mechanisms in the midlatitude $E$ and $F$ regions that are not directly coupled.

Detailed analysis of the Arecibo data involving statistical inverse methods allow the inference of neutral dynamics in the MLT and the thermosphere. Neutral dynamics appears to play a key role in ionospheric instability at middle latitudes. Significant shear flow appears to be nearly ubiquitous in the midlatitude $E$ region. In the patchy $E_{\mathrm{s}}$ layers examined here, the neutral flow was marginally or robustly unstable in the Richardson number sense. In the latter case, instability benefited from regions of less stable lapse rates, which negated the stabilizing effect of buoyancy. The predicted $e$-folding time for the stratum in question was only about 2 min and much shorter than what is anticipated for plasma instability acting alone. Once patchy $E_{\mathrm{s}}$ layers have been induced, fast-growing secondary plasma instabilities should be able to function and produce irregularities on intermediate and small scales.

An interesting feature of the observations presented here is the occurrence of temperature profiles with lapse rates close to the adiabatic value. Adiabatic or near-adiabatic lapse rates are common in the mesosphere where the ambient lapse rate is generally negative. Relatively small perturbations associated with passing wave motions or various types of heating or cooling can easily change the lapse rate, making it more negative and at times getting close to or exceeding the adiabatic value. At altitudes above $100 \mathrm{~km}$ where the background lapse rate is generally isothermal or the temperature increases with height, perturbing the temperature profile sufficiently to reach the adiabatic value is difficult, however. With increasing height in the thermosphere, diffusive effects and radiational cooling effects become more important, but in the lower thermosphere such effects are still relatively small. This suggests that the flow is, at least to a fairly good approximation, nearly adiabatic in that part of the atmosphere. One way to produce an adiabatic layer is therefore to have strong upwelling in the layer, due to mechanical forcing, for example. The motion has to be nearly or entirely vertical since slantwise convection will not lead to an adiabatic lapse rate. The general assumption is often made that the vertical winds in the thermosphere are small because of the highly stable stratification, i.e., the positive or isothermal lapse rates in that part of the atmosphere, but a number of studies have shown that not to be the case. Larsen and Meriwether (2012) summarized a large number of ground-based and in situ vertical wind measurements and found that vertical winds exceeding $10 \mathrm{~m} \mathrm{~s}^{-1}$ are common and often extend over large altitude ranges and over periods of $1 \mathrm{~h}$ or more. With respect to observations specifically from Arecibo, Larsen et al. (2004) presented observations of a number of overturning events in the lower thermosphere, including some from Arecibo. The more recent Hysell et al. (2014a) vertical neutral wind measurements obtained with the Arecibo incoherent scatter radar showed large vertical motions throughout the multi-hour observing period, consistent with the conclusions of Larsen and Meriwether (2012). The forcing responsible for the observed thermospheric vertical winds is not clear, but the occurrence of layers with near-adiabatic lapse rates is to be expected, given that the large vertical winds are there.

In the $F$ region, large-scale plasma irregularities in the bottom side appear to be induced by thermospheric motion with a component parallel to the geomagnetic field in the manner suggested by Crary and Forbes (1986). Once present, the irregularities seed additional irregularities and provide the conditions necessary for plasma convective instability leading to spread $F$. The $e$-folding time for this process appears to be much shorter than that predicted for Perkins instability.

An analytic formulation of the equations for an instability is always attractive, in part because it makes the instability mechanism easier to understand and in part because the stability boundaries can often be identified more easily. The various midlatitude spread $F$ instability theories that have been proposed have either relied entirely on analytic theories or used the analytic theories as a starting point for further numerical calculations. The results presented here are not represented in a simple analytic form but nonetheless show the existence of a robust and fast-growing instability mechanism that appears to account for the observed characteristics of the midlatitude $F$-region plumes that were observed.

\section{Data availability}

The data used for this study are available from the NSF Madrigal Database (http://madrigal.haystack.mit.edu/ madrigal/).

Acknowledgements. This work was supported by awards AGS1360718 and AGS-1360594 from the National Science Foundation to Cornell University and Clemson University. The Arecibo Observatory is part of the National Astronomy and Ionosphere Center which is operated under a cooperative agreement with the National Science Foundation.

The topical editor, D. Pallamraju, thanks two anonymous referees for help in evaluating this paper.

\section{References}

Behnke, R. A.: $F$ layer height bands in the nocturnal ionosphere over Arecibo, J. Geophys. Res., 84, 974-978, 1979.

Bernhardt, P. A.: The modulation of sporadic-E layers by KelvinHelmholtz billows in the neutral atmosphere, J. Atmos. Sol.-Terr. Phy., 64, 1487-1504, 2002.

Bernhardt, P. A., Selcher, C. A., Siefring, C., Wilkens, M., Compton, C., Bust, G., Yamamoto, M., Fukao, S., Takayuki, O., Wakabayashi, M., and Mori, H.: Radio tomographic imaging of sporadic-E layers during SEEK-2, Ann. Geophys., 23, 23572368, doi:10.5194/angeo-23-2357-2005, 2005. 
Bernhardt, P. A., Werne, J., and Larsen, M. F.: Modeling of Sporadic-E Structures from Wind-Driven Kelvin-Helmholtz Turbulence, in: Characterising the Ionosphere, Meeting Proceedings RTO-MP-IST-056, Neuilly-sur-Seine, France, 34, 31-1-31-14, 2006.

Cabrit, B. and Kofman, W.: Ionospheric composition measurement by EISCAT using a global fit procedure, Ann. Geophys., 14(12), 1496-1505, 1996.

Chu, Y. H. and Wang, C. Y.: Interferometry observations of 3dimensional spatial structures of sporadic $E$ using the Chung-Li VHF radar, Radio Sci., 32, 817-832, 1997.

Chu, Y. H., Wang, C. Y., Su, S. L., and Kuong, R. M.: Coordinated sporadic E layer observations made with Chung-Li $30 \mathrm{MHz}$ radar, ionosonde and FORMOSAT-3/COSMIC satellites, J. Atmos. Sol.-Terr. Phy., 73, 883-894, 2011.

Cosgrove, R. B.: Generation of mesoscale $F$ layer structure and electric fields by the combined Perkins and $E_{\mathrm{S}}$ layer instabilities, in simulations, Ann. Geophys., 25, 1579-1601, doi:10.5194/angeo-25-1579-2007, 2007.

Cosgrove, R. B. and Tsunoda, R. T.: A direction-dependent instability of sporadic- $E$ layers in the nighttime midlatitude ionosphere, Geophys. Res. Lett., 29, 1864, doi:10.1029/2002GL014669, 2002.

Cosgrove, R. B. and Tsunoda, R. T.: Instability of the $E-F$ coupled nighttime midlatitude ionosphere, J. Geophys. Res., 109, A04305, doi:10.1029/2003JA010243, 2004.

Crary, D. J. and Forbes, J. M.: The dynamic ionosphere over Arecibo: A theoretical investigation, J. Geophys. Res., 91, 249258, 1986

Didebulidze, G. G. and Lomidze, L. N.: Double atmospheric gravity wave frequency oscillations of sporadic $E$ formed in a horizontal shear flow, Phys. Lett. A, 374, 952-959, 2010.

Duly, T. M., Huba, J. D., and Makela, J. J.: Self-consistent generation of MSTIDs within the SAMI3 numerical model, J. Geophys. Res., 119, 6745-6757, doi:10.1002/2014JA020146, 2014.

Fukao, S., Kelley, M. C., Shirakawa, T., Takami, T., Yamamoto, M., Tsuda, T., and Kato, S.: Turbulent upwelling of the midlatitude ionosphere: 1. Observational results by the MU radar, J. Geophys. Res., 96, 3725-3746, 1991.

Fukao, S., Yamamoto, M., Tsunoda, R. T., Hayakawa, H., and Mukai, T.: The SEEK (Sporadic-E Experiment over Kyushu) campaign, Geophys. Res. Lett., 25, 1761-1764, 1998.

Fukushima, D., Shiokawa, K., Otsuka, Y., and Ogawa, T.: Observation of equatorial nighttime medium-scale traveling ionospheric disturbances in 630-nm airglow images over 7 years, J. Geophys. Res., 117, A10324, doi:10.1029/2012JA017758, 2012.

Gong, Y., Zhou, Q. H., Zhang, S. D., Aponte, N., Sulzer, M., and Gonzalez, S.: Midnight ionosphere collapse at Arecibo and its relationship to the neutral wind, electric field, and ambipolar diffusion, J. Geophys. Res., 117, A08332, doi:10.1029/2012JA017530, 2012.

Haldoupis, C. and Schlegel, K.: Observation of the modified twostream plasma instability in the midlatitude $E$ region ionosphere, J. Geophys. Res., 99, 6219-6226, 1994.

Hamza, A.: Perkins instability revisited, J. Geophys. Res., 104, 22567-22575, doi:10.1029/1999JA900307, 1999.

Hecht, J. H., Liu, A. Z., Bishop, R. L., Clemmons, J. H., Gardner, C. S., Larsen, M. F., Roble, R. G., Swenson, G. R., and Walterscheid, R. L.: An overview of observations of unstable layers during the Turbulent Oxygen Mixing Experiments (TOMEX), J. Geophys. Res., 109, D02S01, doi:10.1029/2002JD003123, 2004.

Helmboldt, J. F., Lazio, T. J. W., Intema, H. T., and Dymond, K. F.: High-precision measurements of ionospheric TEC gradients with the Very Large Array VHF system, Radio Sci., 47, RS0K02, doi:10.1029/2011RS004883, 2012.

Howard, L. N.: Note on a paper by John W. Miles, J. Fluid Mech., 10, 509-512, 1961.

Hysell, D. L., Chau, J. L., and Fesen, C. G.: Effects of large horizonbtal winds on the equatorial electrojet, J. Geophys. Res., 107, 1214, doi:10.1029/2001JA000217, 2002.

Hysell, D. L., Larsen, M. F., and Zhou, Q. H.: Common volume coherent and incoherent scatter radar observations of mid-latitude sporadic E-layers and QP echoes, Ann. Geophys., 22, $3277-$ 3290, doi:10.5194/angeo-22-3277-2004, 2004.

Hysell, D. L., Nossa, E., Larsen, M. F., Munro, J., Sulzer, M. P., Aponte, N., and González, S. A.: Sporadic $E$ layer observations over Arecibo using coherent and incoherent scatter radar: Assessing dynamic stability in the lower thermosphere, J. Geophys. Res., 114, A12303, doi:10.1029/2009JA014403, 2009.

Hysell, D. L., Nossa, E., Larsen, M. F., Munro, J., Smith, S., Sulzer, M. P., and González, S. A.: Dynamic instability in the lower thermosphere inferred from irregular sporadic layers, J. Geophys. Res., 117, A08305, doi:10.1029/2012JA017910, 2012.

Hysell, D. L., Nossa, E., Aveiro, H. C., Larsen, M. F., Munro, J., Sulzer, M. P., and González, S. A.: Fine structure in midlatitude sporadic $E$ layers, J. Atmos. Sol.-Terr. Phy., 103, 16-23, doi:10.1016/j.jastp.2012.12.005, 2013.

Hysell, D. L., Jafari, R., Milla, M. A., and Meriwether, J. W.: Data-driven numerical simulations of equatorial spread $F$ in the Peruvian sector, J. Geophys. Res., 119, 3815-3827 doi:10.1002/2014JA019889, 2014a.

Hysell, D. L., Larsen, M. F., and Sulzer, M. P.: High time and height resolution neutral wind profile measurements across the mesosphere/lower thermosphere region using the Arecibo incoherent scatter radar, J. Geophys. Res., 119, 2345-2358, doi:10.1002/2013JA019621, 2014b.

Kelley, M. C.: On the origin of mesoscale TIDs at midlatitudes, Ann. Geophys., 29, 361-366, doi:10.5194/angeo-29-361-2011, 2011.

Kelley, M. C. and Miller, C. A.: Electrodynamics of midlatitude spread $F$ 3. Electrohydrodynamic waves? A new look at the role of electric fields in thermospheric wave dynamics, J. Geophys. Res., 102, 11539-11547, 1997.

Krall, J., Huba, J. D., Ossakow, S. L., and Joyce, G.: Why do equatorial bubbles stop rising?, Geophys. Res. Lett., 37, L09105, doi:10.1029/2010GL043128, 2010.

Krall, J., Huba, J. D., Ossakow, S. L., Joyce, G., Makela, J. J., Miller, E. S., and Kelley, M. C.: Modeling of equatorial plasma bubbles triggered by non equatorial traveling ionospheric disturbances, Geophys. Res. Lett., 38, L08103, doi:10.1029/2011GL046890, 2011.

Larsen, M. F.: A shear instability seeding mechanism for quasiperiodic radar echoes, J. Geophys. Res., 105, 24931-24940, 2000.

Larsen, M. F.: Winds and shears in the mesosphere and lower thermosphere: Results from four decades of chemical release wind measurements, J. Geophys. Res., 107, 1216, doi:10.1029/2001JA000218, 2002. 
Larsen, M. F. and Meriwether, J. W.: Vertical winds in the thermosphere, J. Geophys. Res., 117, A09319, doi:10.1029/2012JA017843, 2012.

Larsen, M. F., Fukao, S., Yamamoto, M., Tsunoda, R., Igarashi, K., and Ono, T.: The SEEK chemical release experiment: Observed neutral wind profile in a region of sporadic- $E$, Geophys. Res. Lett., 25, 1789-1792, 1998.

Larsen, M. F., Liu, A. Z., Gardner, C. S., Kelley, M. C., Collins, S., Friedman, J., and Hecht, J. H.: Observations of overturning in the upper mesosphere and lower thermosphere, J. Geophys. Res., 109, D02S04, doi:10.1029/2002JD003067, 2004.

Larsen, M. F., Hysell, D. L., Zhou, Q. H., Smith, S. M., Friedman, J., and Bishop, R. L.: Imaging coherent scatter radar, incoherent scatter radar, and optical observations of quasiperiodic structures associated with sporadic $E$ layers, J. Geophys. Res., 112, A06321, doi:10.1029/2006JA012051, 2007.

Layzer, D.: The Turbulence Criterion in Stably Stratified Shear Flow and The Origin of Sporadic E, in: Ionospheric Sporadic $E$, edited by: Smith, E. K. and Matsushita, S., Pergamon Press, Oxford, NY, 258-275, 1962.

Mathews, J. D.: Sporadic E: Current views and recent progress, J. Atmos. Sol.-Terr. Phy., 60, 413-435, 1998.

Miles, J. W.: On the stability of heterogeneous shear flows, J. Fluid Mech., 10, 496-508, 1961.

Miller, E. S., Makela, J. J., and Kelley, M. C.: Seeding of equatorial plasma depletions by polarization electric fields from middle latitudes: Experimental evidence, Geophys. Res. Lett., 36, L18105, doi:10.1029/2009GL039695, 2009.

Miller, K. L. and Smith, L. G.: Incoherent scatter radar observations of irregular structure in mid-latitude sporadic $E$ layers, J. Geophys. Res., 83, 3761-3775, 1978.

Ogawa, T., Nishitani, N., Otsuka, Y., Shiokawa, K., Tsugawa, T., and Hosokawa, K.: Medium-scale traveling ionospheric disturbances observed with the SuperDARN Hokkaido radar, allsky imager, and GPS network and their relation to concurrent sporadic E irregularities, J. Geophys. Res., 114, A03316, doi:10.1029/2008JA013893, 2009.

Otsuka, Y., Onoma, F., Shiokawa, K., Ogawa, T., Yamamoto, M., and Fukao, S.: Simultaneous observations of nighttime mediumscale traveling ionospheric disturbances and $E$ region fieldaligned irregularities at midlatitudes, J. Geophys. Res., 112, A06317, doi:10.1029/2005JA011548, 2007.

Otsuka, Y., Tani, T., Ogawa, T., and Saito, A.: Statistical study of relationship between medium-scale traveling ionospheric disturbance and sporadic $E$ layer activities in summer night over Japan, J. Atmos. Sol.-Terr. Phy., 70, 2196-2202, 2008.

Otsuka, Y., Shiokawa, K., Ogawa, T., Yokoyama, T., and Yamamoto, M.: Spatial relationship of nighttime medium-scale traveling ionospheric disturbances and $F$ region field-aligned irregularities observed with two spaced all-sky airglow imagers and the middle and upper atmosphere radar, J. Geophys. Res., 114, A05302, doi:10.1029/2008JA013902, 2009.

Perkins, F.: Spread $F$ and Ionospheric Currents, J. Geophys. Res., 78, 218-226, 1973.

Press, W. H., Flannery, B. P., Teukolsky, S. A., and Vetterling, W. T.: Numerical Recipes in C, Cambridge University Press, New York, 1988.
Riggin, D., Swartz, W. E., Providakes, J., and Farley, D. T.: Radar studies of long-wavelength waves associated with mid-latitude sporadic E layers, J. Geophys. Res., 91, 8011-8024, 1986.

Saito, S., Yamamoto, M., Hashiguchi, H., and Maegawa, A.: Observation of three-dimensional signatures of quasi-periodic echoes associated with mid-latitude sporadic- $E$ layers by MU radar ultra-multi-channel system, Geophys. Res. Lett., 33, L14109, doi:10.1029/2005GL025526, 2006.

Saito, S., Yamamoto, M., Hashiguchi, H., Maegawa, A., and Saito, A.: Observational evidence of coupling between quasi-periodic echoes and medium scale traveling ionospheric disturbances, Ann. Geophys., 25, 2185-2194, doi:10.5194/angeo-25-21852007, 2007.

Schunk, R. W. and Nagy, A. F.: Ionospheres: Physics, Plasma Physics, and Chemistry, Cambridge University Press, 2004.

Shiokawa, K., Otsuka, Y., Ihara, C., Ogawa, T., and Rich, F. J.: Ground and satellite observations of nighttime medium-scale traveling ionospheric disturbance at midlatitude, J. Geophys. Res., 108, 1145, doi:10.1029/2002JA009639, 2003.

Smith, L. G. and Miller, K. L.: Sporadic- $E$ layers and unstable wind shears, J. Atmos. Sol.-Terr. Phy., 42, 45-50, 1980.

Sulzer, M. P.: A phase modulation technique for a sevenfold statistical improvement in incoherent scatter data-taking, Radio Sci., 21, 737-744, 1986a.

Sulzer, M. P.: A radar technique for high range resolution incoherent scatter autocorrelation function measurements utilizing the full average power of klystron radars, Radio Sci., 21, 1033-1040, $1986 b$.

Sulzer, M. P., Aponte, N., and González, S. A.: Application of linear regularization methods to Arecibo vector velocities, J. Geophys. Res., 110, A10305, doi:10.1029/2005JA011042, 2005.

Suzuki, S., Hosokawa, K., Otsuka, Y., Shiokawa, K., Ogawa, T., Nishitani, N., Shibata, T. F., Koustov, A. V., and Shevtsov, B. M.: Coordinated observations of nighttime medium-scale traveling ionospheric disturbances in 630-nm airglow and $\mathrm{HF}$ radar echoes at midlatitudes, J. Geophys. Res., 114, A07312, doi:10.1029/2008JA013963, 2009.

Swartz, W. E., Kelley, M. C., Makela, J. J., Collins, S. C., Kudeki, E., Franke, S., Urbina, J., Aponte, N., Sulzer, M. P., and Gonzalez, S.: Coherent and incoherent scatter radar observations during intense mid-latitude spread F, Geophys. Res. Lett., 27, 28292832, 2000.

Swisdak, M.: Notes on the dipole coordinate system, Cornell University Library, arXiv:physics/0606044 [physics.space-ph], 2006.

Tsunoda, R. T.: On the coupling of layer instabilities in the nighttime midlatitude ionosphere, J. Geophys. Res., 111, A11304, doi:10.1029/2006JA011630, 2006.

Whitehead, J. D.: The structure of sporadic $E$ from a radio experiment, Radio Sci., 7, 355-358, 1972.

Whitehead, J. D.: Recent work on mid-latitude and equatorial sporadic E, J. Atmos. Sol.-Terr. Phy., 51, 401-424, 1989.

Woodman, R. F., Yamamoto, M., and Fukao, S.: Gravity wave modulation of gradient drift instabilities in mid-latitude sporadic $E$ irregularities, Geophys. Res. Lett., 18, 1197-1200, 1991.

Yamamoto, M., Fukao, S., Woodman, R. F., Ogawa, T., Tsuda, T., and Kato, K.: Mid-latitude $E$ region field-aligned irregularities observed with the MU radar, J. Geophys. Res.-Space, 96, 15943$15949,1991$. 
Yamamoto, M., Fukao, S., Ogawa, T., Tsuda, T., and Kato, S.: A morphological study of mid-latitude $E$-region field-aligned irregularities observed with the MU radar, J. Atmos. Sol.-Terr. Phy., 54, 769-777, 1992.

Yamamoto, M., Fukao, S., Tsunoda, R. T., Pfaff, R., and Hayakawa, H.: SEEK-2 (Sporadic-E Experiment over Kyushu 2) - Project Outline, and Significance, Ann. Geophys., 23, 2295-2305, doi:10.5194/angeo-23-2295-2005, 2005.
Yokoyama, T., Otsuka, Y., Ogawa, T., Yamamoto, M., and Hysell, D. L.: First three-dimensional simulation of the Perkins instability in the nighttime midlatitude ionosphere, Geophys. Res. Lett., 35, L03101, doi:10.1029/2007GL032496, 2008.

Yokoyama, T., Hysell, D. L., Otsuka, Y., and Yamamoto, M.: Threedimensional simulation of the coupled Perkins and $E_{\mathrm{S}}$ layer instabilities in the nighttime midlatitude ionosphere, J. Geophys. Res., 114, A03308, doi:10.1029/2008JA013789, 2009. 